\title{
Dimension Estimates for Certain Sets of Infinite Complex Continued Fractions
}

\author{
J. Neunhäuserer \\ Institut für Mathematik, Technical University Clausthal, Reitstallweg 9, 38640 Goslar, Germany \\ Correspondence should be addressed to J. Neunhäuserer; neunchen@aol.com
}

Received 13 August 2012; Accepted 2 October 2012

Academic Editor: Stefan Siegmund

Copyright (C) 2013 J. Neunhäuserer. This is an open access article distributed under the Creative Commons Attribution License, which permits unrestricted use, distribution, and reproduction in any medium, provided the original work is properly cited.

We prove upper and lower estimates on the Hausdorff dimension of sets of infinite complex continued fractions with finitely many prescribed Gaussian integers. Particulary we will conclude that the dimension of theses sets is not zero or two and there are such sets with dimension greater than one and smaller than one.

\section{Introduction}

Continued fractions were studied in a number of theories since the work of Wallis in the 17th century; see [1]. The first dimensional theoretical perspective on infinite real continued fractions can be found in the work of Jarnik [2], who introduced upper and lower estimates on the Hausdorff dimension of sets of continued fractions with bounded digits. The problem of calculating the dimension of these sets has been addressed by several authors [3-7]. In a resent work Jenkinson and Pollicott provide a fast algorithm to approximate this dimension [8].

Dimension theoretical aspects of infinite complex continued fractions were studied by Mauldin et al. $[9,10]$. They proved that the set of complex continued fractions with arbitrary Gaussian integers from $\mathbb{N}+\mathbb{Z} i$ has Hausdorff dimension greater than one and smaller than two.

We consider here infinite complex continued fractions and ask for the Hausdorff dimension of the set of continued fractions with digits coming from a finite set $A \subset \mathbb{N}[i]$. Using the Moran formula from the theory of iterated function systems [11] we are able to give upper and lower estimates on the Hausdorff dimension of these sets; see Theorem 1. We will show that the dimension of the sets is not zero or two and there are such sets with dimension greater than one and smaller than one; see Corollaries 2 and 3. In addition we provide explicit estimates in selected examples.

\section{Notations, Results, and Examples}

Given a sequence $z_{n} \in \mathbb{C}$ for $n \in \mathbb{N}_{0}$ of Gaussian integers we define the infinite complex continued fraction by

$$
\left[z_{0} ; z_{1}, z_{2}, \ldots\right]:=z_{0}+\frac{1}{1+\left(z_{1} /\left(1+z_{2} \cdots\right)\right)} \in \mathbb{C}
$$

It is well known that every complex number can be represented as an infinite continued fraction of Gaussian integers using the Hurwitz algorithm [12]. Now fix a finite set

$$
A=\left\{a_{j}+b_{j} i \mid a_{j}, b_{j} \in \mathbb{N}, j=1, \ldots, m\right\} \subset \mathbb{N}[i] .
$$

We consider the set of all infinite continued fractions having fractional entries coming from $A$ :

$$
\mathfrak{C}(A):=\left\{\left[0 ; z_{1}, z_{2}, \ldots\right] \mid z_{n} \in A, n \in \mathbb{N}\right\} \subset \mathbb{C} .
$$

Obviously the set $\mathfrak{C}(A)$ is uncountable, and it is a null set with respect to the two-dimension Lebesgue measure (this is immediate from Corollary 2). Thus we are interested in the Hausdorff dimension of this set. Recall $[13,14]$ that the $d$ dimensional Hausdorff measure of a set $\mathfrak{C} \subseteq \mathbb{C}$ is

$$
\begin{gathered}
H^{d}(\mathfrak{C})=\liminf _{\epsilon \rightarrow 0}\left\{\sum_{i=1}^{\infty} \text { diameter }\left(C_{i}\right)^{d} \mid \mathfrak{c} \subseteq \bigcup_{i=1}^{\infty} C_{i},\right. \\
\text { diameter } \left.\left(C_{i}\right)<\epsilon\right\} .
\end{gathered}
$$


The Hausdorff dimension of $\mathfrak{C}$ is given by

$$
\operatorname{dim}_{H} \mathfrak{S}=\sup \left\{d \mid H^{d}(\mathfrak{s})=\infty\right\}=\inf \left\{d \mid H^{d}(\mathfrak{c})=0\right\} .
$$

Now we are able to state our main result on $\operatorname{dim}_{H} \mathfrak{\complement}(A)$.

Theorem 1. For a finite set $A \subset \mathbb{N}[i]$ let $D, d \in \mathbb{R}^{+}$be the unique real numbers fulfilling

$$
\begin{gathered}
\sum_{a+b i \in A}\left(\frac{1}{a^{2}+b^{2}}\right)^{D}=1 \\
\sum_{a+b i \in A}\left(\frac{1}{a^{2}+b^{2}+(1+\sqrt{2}) \max \{a, b\}+1}\right)^{d}=1 .
\end{gathered}
$$

We have

$$
d \leq \operatorname{dim}_{H} \mathfrak{c}(A) \leq D .
$$

Unsing an additionla argument this theorem has the following corollary.

Corollary 2. For all finite sets $A \subset \mathbb{N}[i]$ one has $\operatorname{dim}_{H} \mathfrak{E}(A)<$ 2; on the other hand $\operatorname{dim}_{H} \mathfrak{S}(A)>0$ if $A$ has more than one element.

Proof. Consider

$$
\begin{aligned}
\sum_{a+b i \in A}\left(\frac{1}{a^{2}+b^{2}}\right)^{2} & \leq \sum_{a=1}^{\infty} \sum_{b=1}^{\infty}\left(\frac{1}{a^{2}+b^{2}}\right)^{2} \\
& \leq \frac{1}{4}+\sum_{k=2}^{\infty}(2 k-1)\left(\frac{1}{k^{2}+1}\right)^{2} \\
& \leq-\frac{3}{4}+\sum_{k=1}^{\infty} \frac{2 k-1}{k^{4}}=-\frac{3}{4}+2 \zeta(3)-\zeta(4)<1 .
\end{aligned}
$$

Hence $D<2$. If $A$ has more than one element we have

$$
\sum_{a+b i \in A}\left(\frac{1}{a^{2}+b^{2}+(1+\sqrt{2}) \max \{a, b\}+1}\right)^{0}>1,
$$

hence $d>0$. The result now follows from our theorem.

By a similar argument we get the second corollary.

Corollary 3. There exist finite sets $A \quad \subset \quad \mathbb{N}[i]$ with $\operatorname{dim}_{H} \mathfrak{E}(A)>1$ and there exist such sets with $\operatorname{dim}_{H} \mathfrak{E}(A)<1$.

Proof. Consider

$$
\begin{gathered}
\sum_{a=1}^{\infty} \sum_{b=1}^{\infty}\left(\frac{1}{a^{2}+b^{2}+(1+\sqrt{2}) \max \{a, b\}+1}\right) \\
\geq \sum_{k=1}^{\infty} \frac{2 k-1}{2 k^{2}+(1+\sqrt{2}) k+1} \\
\geq \sum_{k=1}^{\infty}\left(\frac{2}{(4+\sqrt{2}) k}-\frac{1}{2 k^{2}}\right)=\infty .
\end{gathered}
$$

Hence for a suitable choice of $A$ we have

$$
\sum_{a+b i \in A} \frac{1}{a^{2}+b^{2}+(1+\sqrt{2}) \max \{a, b\}+1}>1 .
$$

For this set $A$ we have $d>1$. On the other hand consider $A=\{1+i, 2+i\}$. We have

$$
\sum_{a+b i \in A} \frac{1}{a^{2}+b^{2}}<1
$$

hence $D<1$. The result again follows from our theorem.

We remark that it is possible to deduce the last corollaries from Theorems 1 and 2 of [9] by a few additional arguments. To obtain these results from our main theorem seems to us more transparent.

Our last corollary gives the obvious explicit upper and lower bounds following from Theorem 1.

Corollary 4. For a finite set $A \subset \mathbb{N}[i]$ with cardinality $|A|$ one has,

$$
\begin{aligned}
& \frac{\log (|A|)}{\max _{A} \log \left(a^{2}+b^{2}\right)} \\
& \quad \leq \operatorname{dim}_{H} \mathfrak{E}(A) \\
& \leq \frac{\log (|A|)}{\min _{A} \log \left(a^{2}+b^{2}+(1+\sqrt{2}) \max \{a, b\}+1\right)} .
\end{aligned}
$$

The estimates in this corollary are of course very crude. At the end of this section we will apply Theorem 1 directly to a few examples. Let $A=\{2+2 i, 3+2 i, 3+3 i\}$. The numbers $D$ and $d$ are given by

$$
\begin{aligned}
& \left(\frac{1}{8}\right)^{D}+\left(\frac{1}{13}\right)^{D}+\left(\frac{1}{18}\right)^{D}=1 \\
& \left(\frac{1}{11+2 \sqrt{2}}\right)^{d}+\left(\frac{1}{17+3 \sqrt{2}}\right)^{d}+\left(\frac{1}{22+3 \sqrt{2}}\right)^{d}=1
\end{aligned}
$$

which implies $0.36<\operatorname{dim}_{H} \mathfrak{E}(A)<0.44$, which is an acceptable estimate. If we consider values with small modulus $A=\{1+i, 2+i, 1+2 i\}$ we get

$$
\left(\frac{1}{2}\right)^{D}+2\left(\frac{1}{5}\right)^{D}=1, \quad\left(\frac{1}{4+\sqrt{2}}\right)^{d}+2\left(\frac{1}{8+2 \sqrt{2}}\right)^{d}=1
$$

This gives $0.49<\operatorname{dim}_{H} \mathfrak{S}(A)<0.91$, which is not very good. Let us consider one more example $A=\{3+i, 2+4 i\}$. We get

$$
\left(\frac{1}{10}\right)^{D}+\left(\frac{1}{20}\right)^{D}=1, \quad\left(\frac{1}{14+3 \sqrt{2}}\right)^{d}+\left(\frac{1}{25+4 \sqrt{2}}\right)^{d}=1
$$

and thus $0.21<\operatorname{dim}_{H} \mathfrak{E}(A)<0.27$. As a last example consider $A=\{a+b i \mid a, b=1 \cdots 4\}$. An elementary calculation 
shows that Theorem 1 gives $1<\operatorname{dim}_{H} \mathfrak{F}(A)<1.33$. We like to remark here that it is possible to find an algorithm using thermodynamic formalism that approximates the dimension of $\mathfrak{E}(A)$. We could apply the recent approach of Jekinsion and Pollicott [15] to infinite complex continuous fractions. This approach has the disadvantage that it is not possible to perform necessary calculations without using a computer, which would change the field of our research to computational mathematics.

\section{Proof of the Result}

For $(a, b) \in \mathbb{N}^{2}$ consider transformations $T_{a, b}: \mathbb{C} \mapsto \mathbb{C}$ given by

$$
T_{a, b}(z)=\frac{1}{z+a+b i}
$$

We need three elementary lemmas concerning these transformations to apply the dimension theory of iterated functions systems to the set $\mathfrak{E}(A)$. First we restrict the maps to the open ball $B_{1 / 2}(1 / 2)=\{z \in \mathfrak{F}|| z-1 / 2 \mid<1 / 2\}$.

Lemma 5. For $(a, b) \in \mathbb{N}^{2}$ one has

$$
T_{a, b}\left(B_{1 / 2}\left(\frac{1}{2}\right)\right) \subset B_{1 / 2}\left(\frac{1}{2}\right) \text {. }
$$

Proof. For $I(z)=1 / z$ we have

$$
I\left(B_{r}(z)\right)=B_{r /\left(|z|^{2}-r^{2}\right)}\left(\frac{\bar{z}}{|z|^{2}-r^{2}}\right),
$$

if $|z| \neq r$. Applying the translation with $a+b i$ we obtain

$$
T_{a, b}\left(B_{r}(z)\right)=B_{r /\left(|z+a+b i|^{2}-r^{2}\right)}\left(\frac{\overline{z+a+b i}}{|z+a+b i|^{2}-r^{2}}\right)
$$

if $|z+a+b i| \neq r$. Especially we get

$$
T_{a, b}\left(B_{1 / 2} \frac{1}{2}\right)=B_{1 /\left(2 a+2 a^{2}+2 b^{2}\right)}\left(\frac{1 / 2+a-b i}{a+a^{2}+b^{2}}\right) .
$$

Since $|(1 / 2)+a+b i|>1 / 2$ for $a, b>0$. We have to show the distance of the center of the image to $1 / 2$ plus the radius of the image is less or equal to $1 / 2$. This means

$$
\begin{aligned}
& \left|\frac{1 / 2+a-b i}{a+a^{2}+b^{2}}-\frac{1}{2}\right|+\frac{1}{2 a+2 a^{2}+2 b^{2}} \leq \frac{1}{2} \\
& \Longleftrightarrow\left|a^{2}+b^{2}-a-1+2 b i\right|^{2} \leq\left(a^{2}+b^{2}+a-1\right)^{2} \\
& \Longleftrightarrow\left(a^{2}+b^{2}-a-1\right)^{2}+4 b^{2} \leq\left(a^{2}+b^{2}+a-1\right)^{2} \\
& \Longleftrightarrow 4 a+4 b^{2} \leq 4\left(a^{2}+b^{2}\right) a \Longleftrightarrow 1 \leq a^{2},
\end{aligned}
$$

which is obviously true for a $a \in \mathbb{N}$.

Next we show that the images of the open balls $B_{1 / 2}(1 / 2)$ under different $T_{a, b}$ are disjoint.
Lemma 6. If $\left(a_{1}, b_{1}\right) \neq\left(a_{2}, b_{2}\right)$, one has

$$
T_{a_{1}, b_{1}}\left(B_{1 / 2}(1 / 2)\right) \cap T_{a_{2}, b_{2}}\left(B_{1 / 2}(1 / 2)\right)=\varnothing .
$$

Proof. We have to show that the distance of the balls at hand is bigger or equal to the sum of their radii, that is:

$$
\begin{aligned}
\left|\frac{1 / 2+a_{1}-b_{1} i}{a_{1}+a_{1}^{2}+b_{1}^{2}}-\frac{1 / 2+a_{2}-b_{2} i}{a_{2}+a_{2}^{2}+b_{2}^{2}}\right| \geq & \frac{1}{2 a_{1}+2 a_{1}^{2}+2 b_{1}^{2}} \\
& +\frac{1}{2 a_{2}+2 a_{2}^{2}+2 b_{2}^{2}} .
\end{aligned}
$$

With $d_{1}=a_{1}+a_{1}^{2}+b_{1}^{2}$ and $d_{2}=a_{2}+a_{2}^{2}+b_{2}^{2}$ we have to show

$$
\begin{gathered}
\left|\frac{(1 / 2)+a_{1}-b_{1} i}{d_{1}}-\frac{(1 / 2)+a_{2}-b_{2} i}{d_{2}}\right| \geq \frac{1}{2 d_{1}}+\frac{1}{2 d_{2}} \\
\Longleftrightarrow\left|d_{2}\left(\frac{1}{2}+a_{1}-b_{1} i\right)-d_{1}\left(\frac{1}{2}+a_{2}-b_{2} i\right)\right|^{2} \\
\geq\left(\frac{d_{1}}{2}+\frac{d_{2}}{2}\right)^{2}
\end{gathered}
$$$$
\begin{aligned}
\Longleftrightarrow & \left(\left(\frac{1}{2}+a_{1}\right) d_{2}-\left(\frac{1}{2}+a_{2}\right) d_{1}\right)^{2}+\left(b_{1} d_{2}-b_{2} d_{1}\right)^{2} \\
& \geq\left(\frac{d_{1}}{2}+\frac{d_{2}}{2}\right)^{2}
\end{aligned}
$$$$
\Longleftrightarrow\left(\left(\frac{1}{2}+a_{1}\right)^{2}+b_{1}^{2}\right) d_{2}^{2}-2\left(\frac{1}{2}+a_{1}\right)\left(\frac{1}{2}+a_{2}\right) d_{1} d_{2}
$$$$
+\left(\left(\frac{1}{2}+a_{2}\right)^{2}+b_{2}^{2}\right) d_{1}^{2}-2 b_{1} d_{2} b_{2} d_{1}
$$$$
\geq \frac{d_{1}^{2}}{4}+\frac{d_{1} d_{2}}{2}+\frac{d_{2}^{2}}{4}
$$$$
\Longleftrightarrow d_{1} d_{2}^{2}-\left(\frac{1}{2}+a_{1}+a_{2}+2 a_{1} a_{2}\right) d_{1} d_{2}+d_{2} d_{1}^{2}
$$$$
-2 b_{1} d_{2} b_{2} d_{1} \geq \frac{d_{1} d_{2}}{2}
$$$$
\Longleftrightarrow d_{1}+d_{2}-\left(1+a_{1}+a_{2}+2 a_{1} a_{2}+2 b_{1} b_{2}\right) \geq 0
$$$$
\Longleftrightarrow\left(a_{1}-a_{2}\right)^{2}+\left(b_{1}-b_{2}\right)^{2}-1 \geq 0 \text {. }
$$

This is obviously true under our assumption.

The last lemma contains estimates on the modulus of derivative of the maps on the closed ball $\bar{B}_{1 / 2}(1 / 2)$. 
Lemma 7. For $(a, b) \in \mathbb{N}^{2}$ one has

$$
\begin{gathered}
\max \left\{\left|T_{a, b}^{\prime}(z)\right| z \in \bar{B}_{1 / 2} \frac{1}{2}\right\} \leq \frac{1}{a^{2}+b^{2}}, \\
\min \left\{\left|T_{a, b}^{\prime}(z)\right| z \in \bar{B}_{1 / 2} \frac{1}{2}\right\} \\
\geq \frac{1}{a^{2}+b^{2}+(1+\sqrt{2}) \max \{a, b\}+1} .
\end{gathered}
$$

Proof. For $z \in \bar{B}_{1 / 2}(1 / 2)$ we have

$$
\begin{aligned}
& T_{a, b}^{\prime}(z)=\frac{-1}{(z+a+b i)^{2}} \text { and hence } \\
& \left|T_{a, b}^{\prime}(x+y i)\right|=\frac{1}{(x+a)^{2}+(y+b)^{2}} .
\end{aligned}
$$
that

Now the first estimate is obvious. For the second part note

$$
\begin{aligned}
& \max \left\{(x+a)^{2}+(y+b)^{2} \mid z=x+i y \in \bar{B}_{1 / 2}\left(\frac{1}{2}\right)\right\} \\
& =\max \left\{(x+a)^{2}+(y+b)^{2} \mid\left(x-\frac{1}{2}\right)^{2}\right. \\
& \left.+y^{2} \leq \frac{1}{4}, x, y \in \mathbb{R}\right\} \\
& =a^{2}+b^{2}+\max \left\{x+2 x a+2 y b \mid y^{2} \leq x-x^{2},\right. \\
& \left.x \in[0,1], y \in\left[\frac{-1}{2}, \frac{1}{2}\right]\right\} \\
& \leq 1+a^{2}+b^{2}+2 \max \left\{x a+y b \mid y^{2} \leq x-x^{2},\right. \\
& \left.x \in[0,1], y \in\left[\frac{-1}{2}, \frac{1}{2}\right]\right\} \\
& \leq 1+a^{2}+b^{2} \\
& +2 \max \{a, b\} \max \left\{x+y \mid y^{2} \leq x-x^{2},\right. \\
& \left.x \in[0,1], y \in\left[\frac{-1}{2}, \frac{1}{2}\right]\right\} \\
& \leq 1+a^{2}+b^{2}+(1+\sqrt{2}) \max \{a, b\}
\end{aligned}
$$

using the approach of Lagrange in the last estimate. This implies the result.

Given a finite set $A \subset \mathbb{N}[i]$ consider the iterated function system (IFS) in the sense of Hutchinson [16]:

$$
\left(\bar{B}_{1 / 2}\left(\frac{1}{2}\right),\left\{T_{a, b} \mid a+b i \in A\right\}\right) .
$$
that is:

By Lemma 5 this IFS is well defined with attractor $\mathfrak{C}(A)$,

$$
\mathfrak{E}(A)=\bigcup_{a+b i \in A} T_{a, b}(\mathfrak{S}(A))
$$

By Lemma 6 the IFS fulfills the open set condition, first introduced by Moran [11]. Moreover by Lemma 7 we have

$$
\begin{aligned}
& \frac{\left|z_{1}-z_{2}\right|}{a^{2}+b^{2}+(1+\sqrt{2}) \max \{a, b\}+1} \\
& \leq\left|T_{a, b}\left(z_{1}\right)-T_{a, b}\left(z_{2}\right)\right| \leq \frac{\left|z_{1}-z_{2}\right|}{a^{2}+b^{2}}
\end{aligned}
$$

for all $z_{1}, z_{2} \in \bar{B}_{1 / 2}(1 / 2)$ and all $a+b i \in A$. Now Theorem 1 is a direct application of Theorem 8.8 of Falconer [17], a wellknow result in the dimension theory of IFS, which goes back to Moran [11].

\section{References}

[1] J. Havil, Gamma: Exploring Euler's Constant, Princeton University Press, Princeton, NJ, USA, 2003.

[2] V. Jarnik, "Diophantischen Approximationen und Hausdorffsches Mass," Matematicheskii Sbornik, vol. 36, pp. 371-382, 1929.

[3] I. J. Good, "The fractional dimension of continued fractions," Proceedings of the Cambridge Philosophical Society, vol. 37, pp. 199-208, 1941.

[4] R. T. Bumby, "Hausdorff dimension of Cantor sets," Journal für die reine und angewandte Mathematik, vol. 331, pp. 192-206, 1982.

[5] R. T. Bumby, Hausdorff Dimension of Sets Arising in Number Theory, Lecture Notes in Mathematics, vol. 1135, Springer, New York, NY, USA, 1985.

[6] D. Hensley, "The Hausdorff dimensions of some continued fraction cantor sets," Journal of Number Theory, vol. 33, no. 2, pp. 182-198, 1989.

[7] D. Hensley, "A polynomial time algorithm for the Hausdorff dimension of continued fraction Cantor sets," Journal of Number Theory, vol. 58, no. 1, pp. 9-45, 1996.

[8] O. Jenkinson and M. Pollicott, "Computing the dimension of dynamically defined sets: E2 and bounded continued fractions," Ergodic Theory and Dynamical Systems, vol. 21, no. 5, pp. 1429-1445, 2001.

[9] R. J. Gardner and R. D. Mauldin, "On the Hausdorff dimension of a set of complex contiuned fractions," Illinois Journal of Mathematics, vol. 27, no. 2, pp. 334-345, 1983.

[10] R. D. Mauldin and M. Urbański, "Dimensions and measures in infinite iterated function systems," Proceedings of the London Mathematical Society, vol. 73, no. 1, pp. 105-154, 1996.

[11] P. Morna, "Additiv funktions of Intervalls and Hausdorff dimension," Proceedings of the Cambridge Philosophical Society, vol. 42, pp. 15-23, 1946.

[12] A. Hurwitz, "Uber die Entwicklung Complexer Grössen in Kettenbruche," Acta Mathematica, vol. 11, pp. 187-200, 1895.

[13] K. Falconer, Fractal Geometry-Mathematical Foundations and Applications, Wiley, New York, NY, USA, 1990.

[14] Y. A. Pesin, Dimension Theory in Dynamical Systems-Contemplary Views and Applications, University of Chicago Press, 1997.

[15] O. Jenkinson and M. Pollicott, "Calculating Hausdorff dimension of Julia sets and Kleinian limit sets," American Journal of Mathematics, vol. 124, no. 3, pp. 495-545, 2002. 
[16] J. E. Hutchinson, "Fractals and self-similarity," Indiana University Mathematics Journal, vol. 30, pp. 271-280, 1981.

[17] K. Falconer, Techniques in Fractal Geometry, Wiley, New York, NY, USA, 1997. 


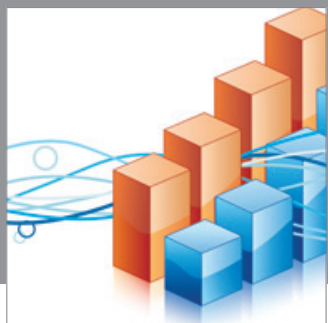

Advances in

Operations Research

mansans

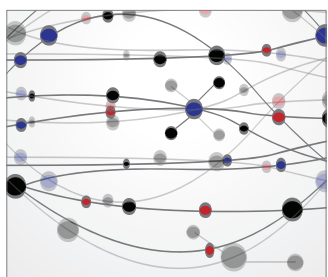

The Scientific World Journal
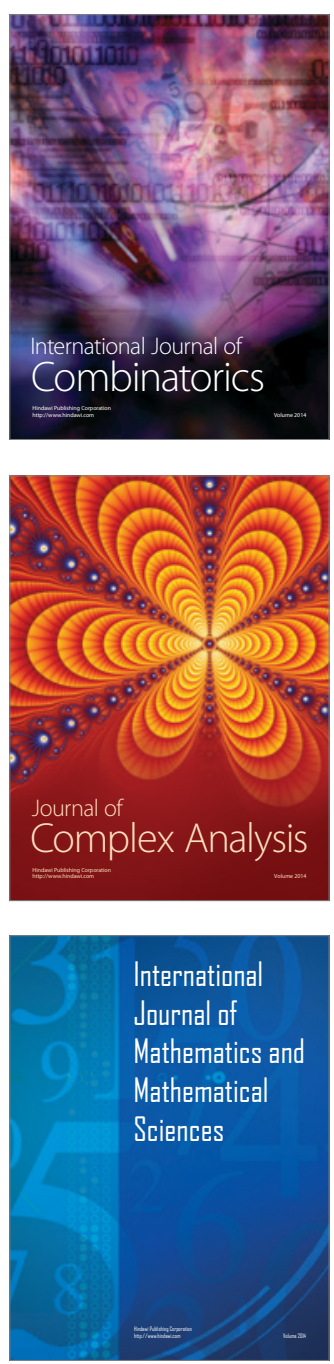
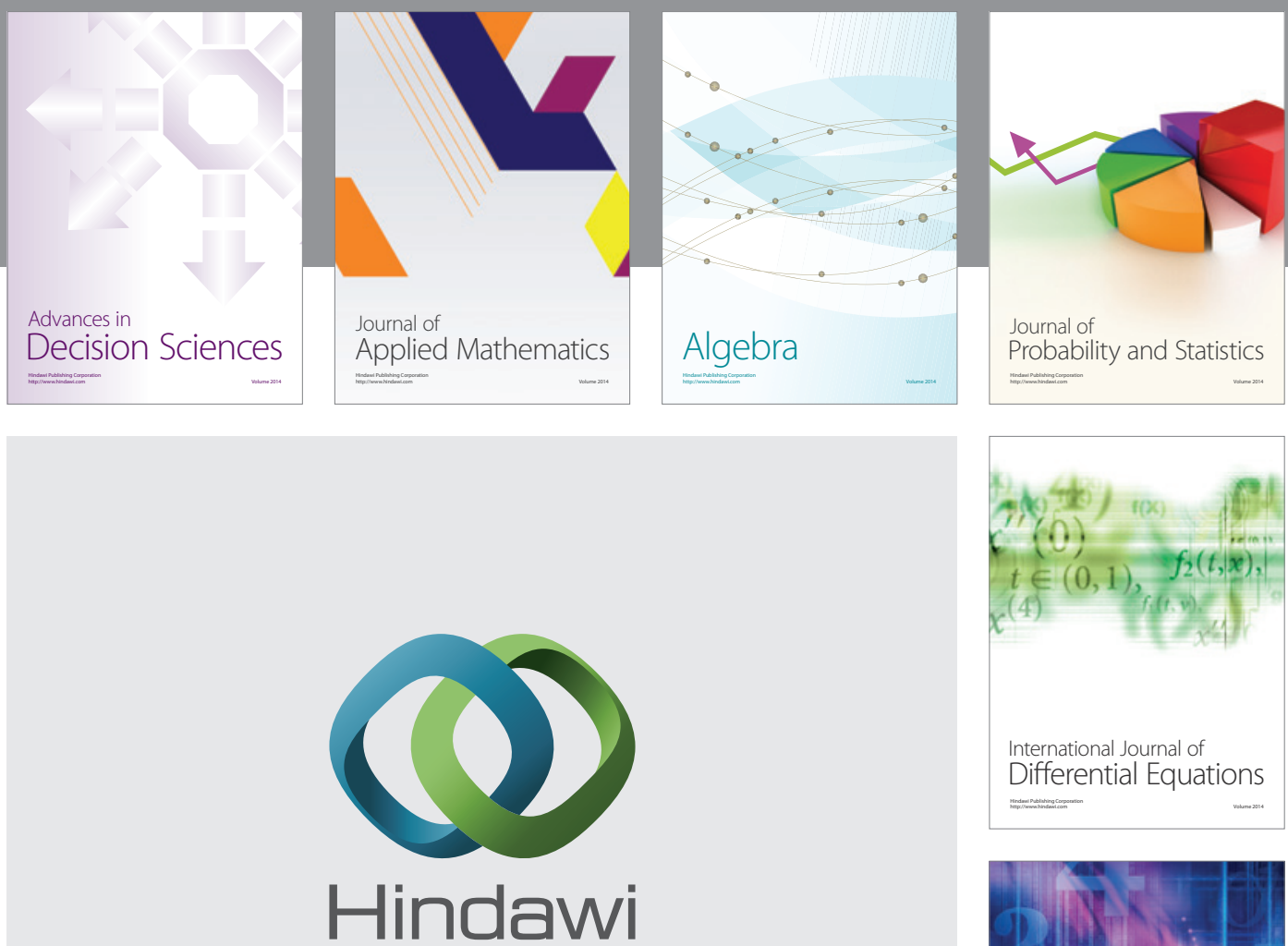

Submit your manuscripts at http://www.hindawi.com
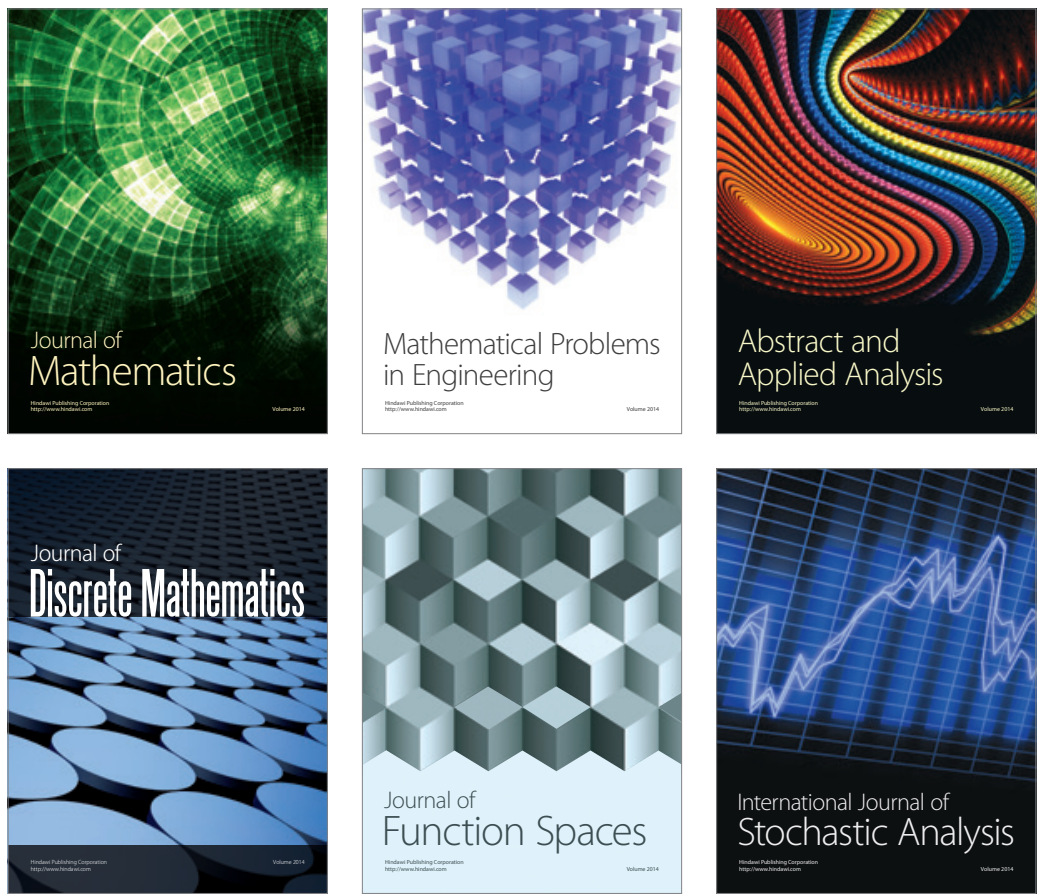

Journal of

Function Spaces

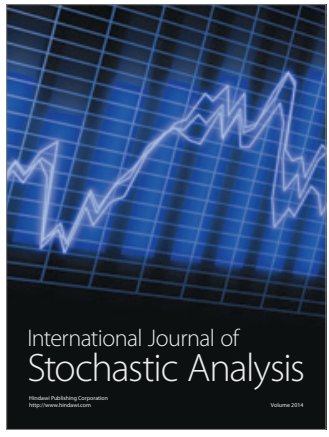

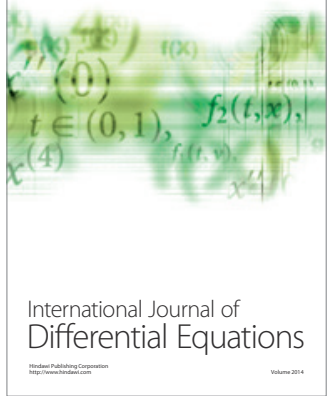
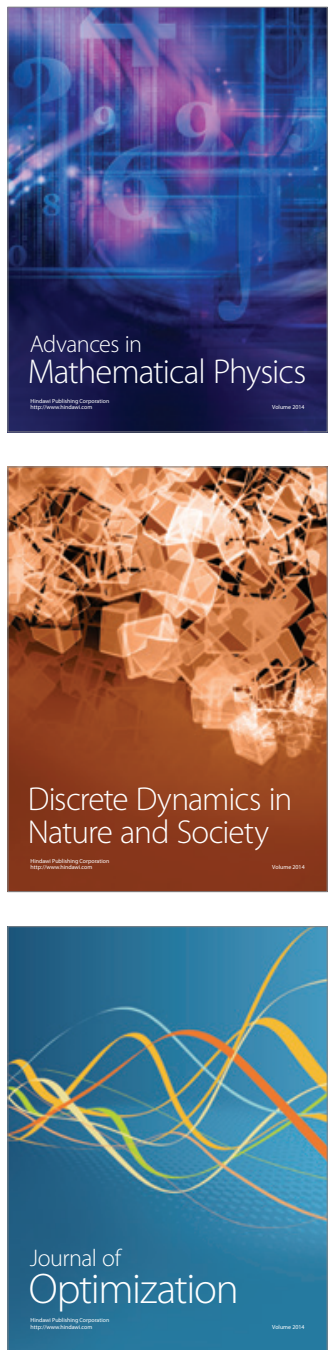